\title{
Pulsed laser modulation for adaptive excitation of multiphoton neuronal imaging in vivo (Withdrawal Notice)
}

Yujie Xiao, Bo Li

Yujie Xiao, Bo Li, "Pulsed laser modulation for adaptive excitation of multiphoton neuronal imaging in vivo (Withdrawal Notice)," Proc. SPIE 11902, Real-time Photonic Measurements, Data Management, and Processing VI, 119020C (9 October 2021); doi: 10.1117/12.2601121

SPIE. Event: SPIE/COS Photonics Asia, 2021, Nantong, Jiangsu, China 


\section{Pulsed laser modulation for adaptive excitation of multiphoton neuronal imaging in vivo (Withdrawal Notice)}

Yujie Xiao, Bo Li

Fudan Univ. (China)

Proceedings Volume 11902, SPIE/COS Photonics Asia; $119020 \mathrm{C}$ (2021) https://doi.org/10.1117/12.2601121

Event: SPIE/COS Photonics Asia

Online Publication Date: 9 October 2021

Withdrawn from Publication: 13 October 2021

Publisher's Note: This paper, originally published on 9 October 2021, was withdrawn on 13 October 2021 per author request. 